\title{
Controlling Hydrogenation of Graphene on Transition Metals
}

\author{
M. L. Ng, ${ }^{\dagger, \ddagger}$ R. Balog,${ }^{\S}$ L. Hornekær, ${ }^{\S}$ A. B. Preobrajenski, ${ }^{*, \$}$ N. A. Vinogradov,, \\ N. Mårtensson, ${ }^{\dagger,}$ and K. Schulte*,* \\ Department of Physics, Uppsala University, Box 530, 75121 Uppsala, Sweden; MAX-lab, Lund University, \\ Box 118, 22100 Lund, Sweden; and Department of Physics and Astronomy and Interdisciplinary Nanoscience \\ Center (iNano), Aarhus University, 8000 Aarhus C, Denmark
}

Received: July 9, 2010; Revised Manuscript Received: September 9, 2010

A monatomic layer of graphite (MG or graphene) adsorbed on the (111) faces of transition metals Pt, Ir, and $\mathrm{Ni}$, has been employed for controlling the atomic hydrogen adsorption site selectivity and the amount of hydrogen adsorbed upon saturation. The variations in the graphene-metal chemical bonding caused by hydrogenation and the values of saturated hydrogen coverage have been studied by X-ray photoemission and $\mathrm{X}$-ray absorption spectroscopy. The hydrogenation of the graphene/metal systems has also been compared to the hydrogen adsorption on highly oriented pyrolytic graphite under the same experimental conditions. It has been found that graphene adsorption on the transition metal substrates can drastically enhance the hydrogen uptake values. The highest values have been observed for MG/Ir(111), less for MG/Pt(111), even less for MG/Ni and the least for the adsorption on bulk graphite. The high level of $\mathrm{H}$ coverage on MG/Ir and MG/Pt has been assigned to the preferential $\mathrm{H}$ adsorption on the more bonding patches (pores) of the MG/metal coincidence lattice. This adsorption creates unpaired electrons which contribute to a strengthening of the graphene-metal bonds. In this way, the densest possible graphane-like patches can be formed on MG/Pt and $\mathrm{MG} / \mathrm{Ir}$. On the MG/Ni interface the formation of graphane is obstructed by the strong interfacial bonding.

\section{Introduction}

Graphene or monolayer graphite (MG) offers tantalizing opportunities in the relentless race toward smaller and faster electronics due to its two-dimensional structure and excellent electron mobility. ${ }^{1,2}$ A controllable band gap opening, however, is a precondition, which needs to be achieved before graphene can be utilized in e.g. carbon-based electronics. In addition, graphene is a suitable material for chemical vapor sensing, since its electrical properties are very sensitive to adsorption of various molecules. ${ }^{3}$ It has been shown that electronic changes in graphene can be realized by means of controllable chemical functionalization. ${ }^{4}$ In particular, hydrogenation of graphene offers an elegant route toward tailoring electronic (metalsemiconductor transition) and magnetic (nonmagnetic-ferromagnetic) properties of graphene,$^{5-10}$ as well as providing the opportunity for hydrogen storage. ${ }^{11,12}$

Experimental investigations of hydrogenation of graphene have revealed very different structural behavior on supported and free-standing graphene. On $\mathrm{SiC}$ supported graphene, the adsorption of individual $\mathrm{H}$ atoms results in the formation of coadsorbed pairs of $\mathrm{H}$ atoms on either neighboring $\mathrm{C}$ atoms (1,2-dihydrogenated ring, ortho-dimer) or opposite $\mathrm{C}$ atoms $(1,4-$ dihydrogenated ring, para-dimer) in the graphene ring. ${ }^{13} \mathrm{Al}-$ though at very low coverage dimers preferentially form on the protruding areas of a graphene sheet modulated by the underlying $6 \times 6$ reconstruction, with increasing coverage, of hydrogen atoms tend to cluster in a rather disordered manner. ${ }^{13}$ Therefore, the hydrogen structures formed on the SiC supported graphene very much resemble those known on the graphite

* To whom correspondence should be addressed: E-mail: alexeip@ maxlab.lu.se; karina.schulte@maxlab.lu.se.

† Department of Physics, Uppsala University.

* MAX-lab, Lund University.

$\S$ Department of Physics and Astronomy, Aarhus University. surface. ${ }^{14}$ However, results from experiments with hydrogen treated free-standing graphene indicate the formation of a graphane structure. ${ }^{8}$ Here, a dense packing of one hydrogen per carbon placed on alternating sides of the sheet for adjacent carbon atoms forms a highly stable regular arrangement through $s p^{3}$ hybridization. ${ }^{11}$ Recently, similar $s p^{3}$ configuration has been observed on $\operatorname{Ir}(111)$ supported graphene, where a $\mathrm{C}-\mathrm{H}$ bond at the $\mathrm{MG}-\mathrm{Ir}$ interface is substituted by the formation of a strong C-Ir bond. ${ }^{10}$ Moreover, the hydrogenation of graphene on $\operatorname{Ir}(111)$ generates a highly regular hydrogen pattern, giving rise to a large gap opening at the Fermi level. From these experimental findings, it is clear that the interaction of hydrogen with graphene strongly depends on the underlying substrate and on whether only one or both sides of the graphene sheet are chemically active. An important question is then, how the strength of the chemical interaction between $\mathrm{MG}$ and the underlying substrate modifies the hydrogen reactivity. This issue can be addressed by studying hydrogenation of graphene on several different transition metal (TM) substrates with varying strength of the interfacial interaction. In addition, graphene layers formed on transition metals are often naturally and periodically nanostructured due to a combination of the MG/TM lattice mismatch and a certain strength of chemical bonding ${ }^{15,16}$ thus, offering nanoscale regions with different hydrogen reactivity in the same MG/TM system.

In the present work, we compare the hydrogenation processes for graphene grown on $\operatorname{Pt}(111), \operatorname{Ir}(111)$, and $\mathrm{Ni}(111)$. The strength of interaction between graphene and the substrates is increasing in the series $\mathrm{Pt}-\mathrm{Ir}-\mathrm{Ni}^{15}$ providing a possibility to correlate it with the hydrogen uptake. For comparison, we present the case of highly oriented pyrolytic graphite (HOPG) hydrogenated under the same conditions as the MG/TM interfaces. Since there is no significant chemical interaction between the atomic layers in HOPG, this reference experiment 
allows us to demonstrate the general impact of the metal substrates on the hydrogenation process. The characterization of the samples is performed by a combination of core-level photoelectron spectroscopy (PES), near-edge X-ray absorption fine structure (NEXAFS) spectroscopy and low energy electron diffraction (LEED). With these techniques we reveal the influence of the MG/TM interface reactivity on the total hydrogen uptake and study site selectivity of the hydrogen adsorption.

\section{Experimental Section}

All samples were prepared in situ at beamline D1011, MAXlab, Lund University. The single crystal substrates Pt(111), $\mathrm{Ir}(111)$, and $\mathrm{Ni}(111)$ were cleaned with several cycles of $\mathrm{Ar}^{+}$ sputtering, annealing and oxygen treatment (whenever necessary). A single monolayer of graphene was prepared by thermal cracking of propylene molecules on the hot crystal surface, i.e., $650{ }^{\circ} \mathrm{C}$ on $\mathrm{Pt}, 780{ }^{\circ} \mathrm{C}$ on $\mathrm{Ir}$, and $670{ }^{\circ} \mathrm{C}$ on $\mathrm{Ni}$ for $30 \mathrm{~min}$ in the propylene partial pressure of $3-5 \times 10^{-8}$ mbar. The presence of MG was checked by the core level intensity ratios in PES and confirmed by LEED patterns characteristic for just one atomic layer of graphene. The HOPG sample was cleaved in situ and annealed to $600{ }^{\circ} \mathrm{C}$. The samples were hydrogenated at room temperature (RT) by atomic hydrogen from an Omicron EFM H source oriented normal to the substrate surface. To reach hydrogen saturation, we operated the source at $T=2200 \mathrm{~K}$ $(30 \mathrm{~mA}$ at $1 \mathrm{kV})$ in a hydrogen gas partial pressure of $1.0 \times$ $10^{-7} \mathrm{mbar}$ for $15 \mathrm{~min}$. The saturation level was confirmed by PES, i.e., no further changes occurred to the spectra with increasing $\mathrm{H}$ exposure at this source temperature. Furthermore, the saturation level for MG on Pt, Ir, and HOPG was also checked at higher source temperature, i.e., $2600 \mathrm{~K}$, but no perceptible changes could be observed. Only the samples fully hydrogenated to saturated coverage are discussed throughout this work. All samples were characterized by core level PES, NEXAFS, and LEED. The LEED results are not discussed in the following because no ordered surface symmetry was detected, implying that $\mathrm{H}$ adsorption occurred randomly. The C 1s NEXAFS spectra were measured in partial electron yield (PEY) mode $(U=-100 \mathrm{~V})$ for higher surface sensitivity. The photon energy resolution for NEXAFS at the C K-edge was set to $75 \mathrm{meV}$. The PE spectra were accumulated in normal emission at a resolution of $125 \mathrm{meV}$. All NEXAFS spectra were normalized to spectra from the clean metal surfaces. The core level PE spectra were measured relative to the Fermi level and deconvoluted using the FitXPS software. ${ }^{17}$

\section{Results and Discussion}

Figure 1 depicts the C1s NEXAFS of pristine (black curves) versus $\mathrm{H}$-saturated (red curves) $\mathrm{HOPG}$ and $\mathrm{MG}$ on $\mathrm{Pt}$, Ir, and Ni. Prior to hydrogen treatment, we observe a common trend of increasingly perturbed electronic structure at the $\mathrm{C} \mathrm{K}$-edge in the sequence of $\mathrm{HOPG}-\mathrm{MG} / \mathrm{Pt}-\mathrm{MG} / \mathrm{Ir}-\mathrm{MG} / \mathrm{Ni}{ }^{15}$ The original features $\mathrm{A}, \mathrm{B}$, and $\mathrm{C}$, which are signatures of the $\pi^{*}$, $\sigma_{1}^{*}$, and $\sigma_{2}^{*}$ states respectively, are most distinctive in the spectrum of clean HOPG (Figure 1a). For graphene on the $5 \mathrm{~d}$ metals, peak $\mathrm{A}$ has gained an additional shoulder $\mathrm{A}_{1}$ while peaks $\mathrm{B}$ and $\mathrm{C}$ have become broader, although the overall spectral shape is preserved (Figure 1b,c). For the MG/Ni case (Figure 1d), another peak $A_{2}$ has appeared, whereas peaks $B$ and $C$ are completely smeared out. This signifies that the $\mathrm{MG}-\mathrm{Ni}$ interaction is of a strong chemical nature. Therefore, the interaction between the top layer graphene and the substrate is growing from physisorption (HOPG) to weak chemisorption (MG/Pt and

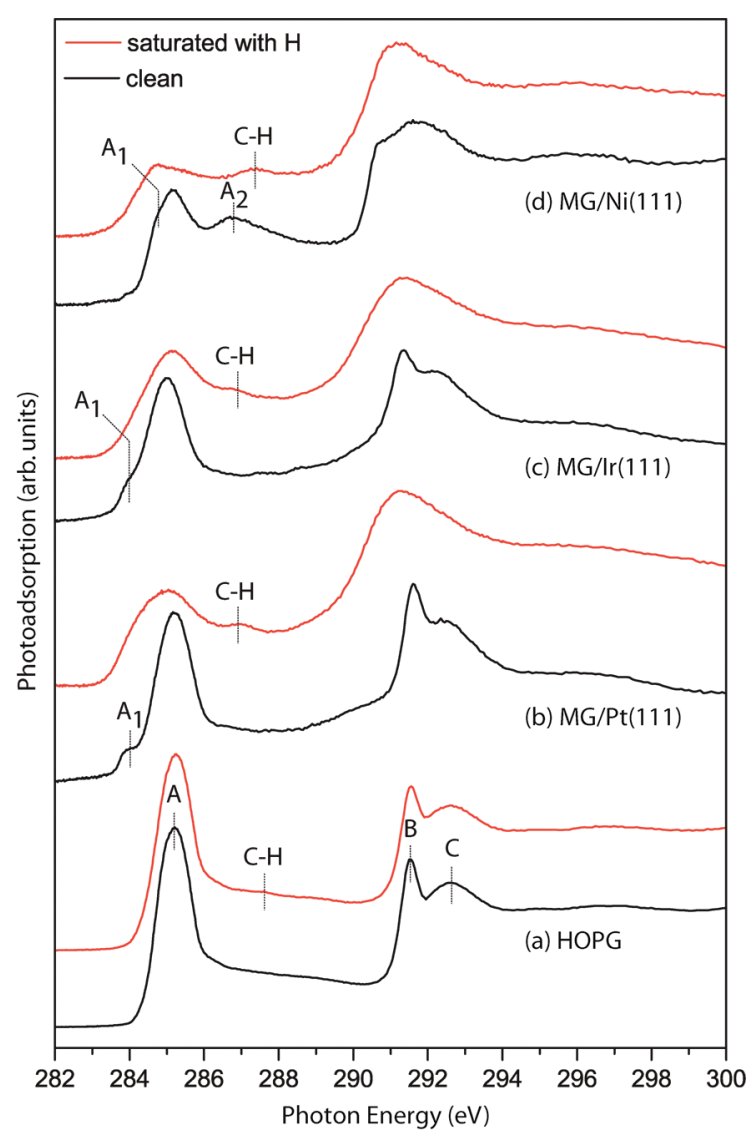

Figure 1. The C 1s NEXAFS spectra from the pristine (black curves) and the hydrogen saturated (red curves) samples. The angle of incidence is $50{ }^{\circ} \mathrm{C}$ in all cases. (a) HOPG, (b) MG on Pt(111), (c) MG on $\operatorname{Ir}(111)$, and (d) MG on $\mathrm{Ni}(111)$.

$\mathrm{MG} / \mathrm{Ir}$ ) and finally to strong chemisorption (MG/Ni). For the lattice-mismatched systems (MG/Pt and MG/Ir), the periodic alternation of favorable and unfavorable adsorption sites results in a weak periodic corrugation of graphene with a period of several nanometers. In this work, we will refer to the low lying (more bonding) parts as "pores" and to the elevated (less bonding) parts as "wires". Since the MG-Ir bond is stronger than that of $\mathrm{MG}-\mathrm{Pt}$, the corrugation of graphene is somewhat more pronounced on $\operatorname{Ir}(111)$ than on $\operatorname{Pt}(111) .{ }^{15}$

Upon hydrogenation, the spectrum of HOPG (Figure 1a) is virtually unchanged, except for the appearance of a minor peak at $287.5 \mathrm{eV}$ commonly associated with the $\mathrm{C}-\mathrm{H}$ bonds in the C 1s NEXAFS of hydrocarbons. ${ }^{18}$ The robustness of the $\mathrm{C} 1 \mathrm{~s}$ NEXAFS shape of HOPG upon hydrogenation is due to the fact that atomic hydrogen does not intercalate into graphite, but chemisorbs on the topmost layer only. ${ }^{14,19}$ Since several graphitic layers contribute to the signal, it is hard to determine how much the electronic structure of the topmost layer is affected by hydrogenation. In contrast, adsorption of hydrogen on the MG/ TM systems results in an obvious change of the spectral shape (Figure 1b,c) indicating a significant modification of the graphene electronic structure. All features are broadened, the $\pi^{*}$ state intensity is lowered, the $\mathrm{C}-\mathrm{H}$ peak is more pronounced, and the energy separation between the $\pi^{*}$ and $\sigma^{*}$ manifolds is reduced for the case of $\mathrm{Pt}$ and Ir. All of these changes indicate that the carbon atoms form chemical bonds with hydrogen. The reduced $\pi^{*}-\sigma^{*}$ separation for the H/MG/Pt and H/MG/Ir interfaces as compared to their non-hydrogenated counterparts implies weakening of the in-plane $\mathrm{C}-\mathrm{C}$ bonds upon hydrogenation. This is understandable because hydrogen reacts with the 


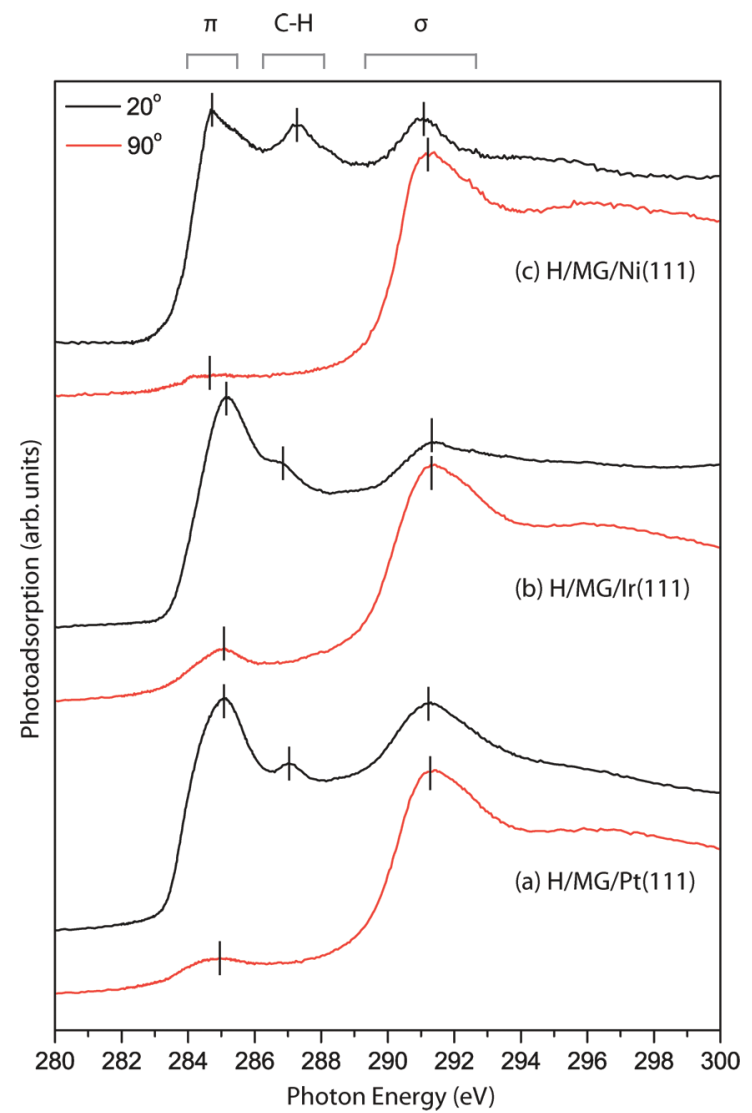

Figure 2. Angle-resolved C 1s NEXAFS spectra of hydrogenated graphene on (a) $\operatorname{Pt}(111)$, (b) $\operatorname{Ir}(111)$, and (c) $\mathrm{Ni}(111)$.

basal plane which results in double bond breakage. For MG/ $\mathrm{Ni}$, the in-plane $\mathrm{C}-\mathrm{C}$ bonds are already weakened in the pristine layer due to the strong chemisorption. In none of the MG layers does the $\pi^{*}$ state intensity vanish entirely, signifying the presence of a certain amount of unhydrogenated graphene patches in all cases.

The orientation of the $\mathrm{C}-\mathrm{H}$ bonds relative to the surface can be estimated by recording the NEXAFS spectra at different angles. In Figure 2, we show angle dependent $\mathrm{C}$ 1s NEXAFS spectra from different $\mathrm{H} / \mathrm{MG} / \mathrm{TM}$ interfaces measured with $\theta$ at $20^{\circ} \mathrm{C}$ (grazing incidence) and $90^{\circ}$ (normal incidence), where $\theta$ is the angle between the polarization vector of the light and the surface normal. From these spectra, it is apparent that the $\mathrm{C}-\mathrm{H}$ bonds are oriented perpendicular to the graphene plane for all three interfaces, as no signature of these bonds is detected at normal incidence. A small tilt of the $\mathrm{C}-\mathrm{H} \sigma^{*}$ bonds accompanying the formation of $\mathrm{H}-\mathrm{H}$ dimers cannot be entirely excluded, but it is below the detection limit in our measurements. It should be noted, however, that if the graphene flakes would have been too small (or their defect density too large), the contribution from flat lying $\mathrm{C}-\mathrm{H}$ bonds at the flake boundaries would become important and would also result in $\mathrm{C}-\mathrm{H}$ related intensity at normal incidence.

Information about the exact hydrogen uptake values and the $\mathrm{H}$ adsorption positions can be obtained by analyzing highresolution core level PES data. Figure 3 shows the carbon core level photoemission of the HOPG and MG layers on Ir and Pt before and after hydrogenation to saturated coverage. In the schematic drawings, the large gray circles represent metal atoms while the medium (colored) circles represent carbon atoms with various chemical environments in the graphene layer. Each color symbolizes a specific chemical state related to the components in the PE spectra. The small black circles are hydrogen atoms.
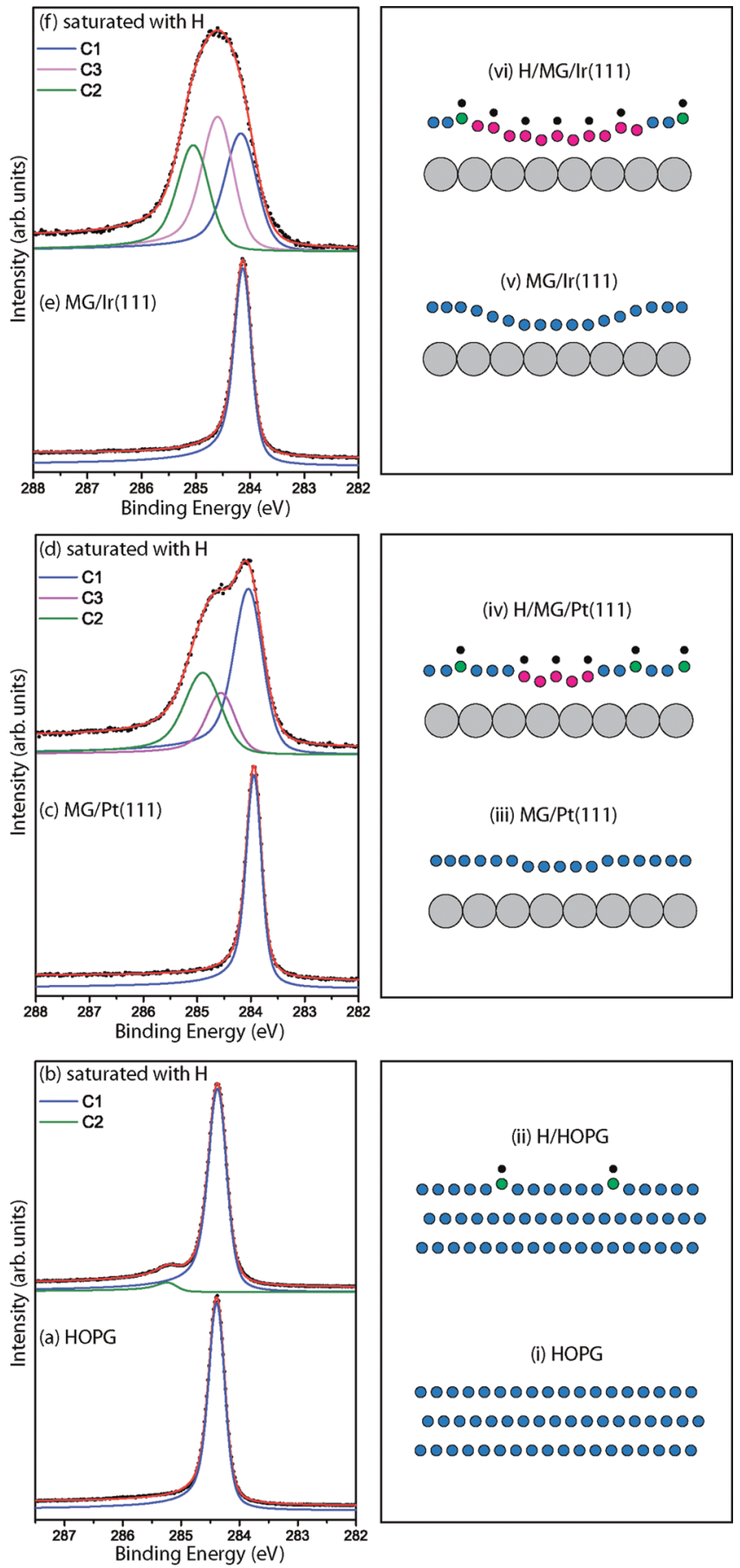

Figure 3. $\mathrm{C} 1 \mathrm{~s}$ PES taken with $\mathrm{h} v=400 \mathrm{eV}$ (left) and the respective schematic drawings (right) of pristine and hydrogen saturated HOPG and MG on $\operatorname{Pt}(111)$ and $\operatorname{Ir}(111)$ [from bottom to top].

For clean HOPG (Figure 3a), the core level spectrum consists of a single component at $284.4 \mathrm{eV}$, accounting for the carbon species with the $s p^{2}$ bonds. Similarly, the spectra for the weakly hybridized graphene on Pt and Ir (Figure 3c,e) have only one component each despite the weak corrugation caused by the lattice mismatch. ${ }^{15}$ The degree of graphene corrugation on $\mathrm{Pt}$ is slightly lower than on Ir ${ }^{15}$ as shown schematically in Figure 3(iii,v). On Ni, MG is strongly adsorbed and lattice matched, implying no nanoscale corrugation. Instead, the $\mathrm{C}$ atoms are located in an alternating manner on top of the $\mathrm{Ni}$ atoms and in fcc hollow sites, thus giving rise to an atomic scale rippling ${ }^{20,21}$ (see Figure 5, i).

In general, the hydrogenation process can be categorized according to the sample reactivity, namely nonactivated graphene 
TABLE 1: A Summary of Binding Energies for All Components in the $\mathrm{C}$ 1s PE Spectra

\begin{tabular}{clc}
\hline surface & component & BE $(\mathrm{eV})$ \\
\hline H/MG/Ni & dissolved C & 283.2 \\
& $\mathrm{c} 3$ & 284.2 \\
& $\mathrm{c} 1$ & 284.7 \\
& $\mathrm{c} 2$ & 285.3 \\
MG/Ni & dissolved C & 283.2 \\
& $\mathrm{c} 1$ & 284.8 \\
H/MG/Ir & $\mathrm{C} 1$ & 284.1 \\
& $\mathrm{C} 3$ & 284.6 \\
MG/Ir & $\mathrm{C} 2$ & 285.0 \\
H/MG/Pt & $\mathrm{C} 1$ & 284.1 \\
& $\mathrm{C} 1$ & 284.0 \\
MG/Pt & $\mathrm{C} 3$ & 284.5 \\
H/HOPG & $\mathrm{C} 2$ & 284.9 \\
& $\mathrm{C} 1$ & 283.9 \\
HOPG & $\mathrm{C} 1$ & 284.4 \\
\end{tabular}

(HOPG), activated and weakly corrugated graphene (on Pt and Ir) and strongly activated and lattice matched graphene (on $\mathrm{Ni}$ ). For the HOPG sample, the contribution of the topmost layer to the total $\mathrm{C} 1 \mathrm{~s}$ signal is determined as $1-\exp (-d / \lambda)$, where $d$ $=3.2 \AA$ is the interlayer distance and $\lambda=4.5 \AA$ is the inelastic mean free path of the electrons, ${ }^{22}$ giving approximately $50 \%$. The peak at $E_{\mathrm{B}}=285.2 \mathrm{eV}$ (Figure 3(b)[green]) is interpreted as the $\mathrm{C}-\mathrm{H}$ bond, in agreement with ref 19 . By comparing the area under the $\mathrm{C}-\mathrm{H}$ and the $\mathrm{C}=\mathrm{C}$ components, we obtain a rather low saturated hydrogen uptake value of only $12 \%$ for HOPG, which is much lower than the value reported previously in ref 19 (37\%). Further increase in the source temperature did not result in the increased $\mathrm{H}$ coverage in our experiments. In part, this discrepancy can be due to a different defect density for different HOPG samples or due to differences in sample preparation.

For the weakly bound MG/Ir and MG/Pt interfaces, the hydrogenation results in a redistribution of the spectral intensity in favor of the new components at higher BE (Figure 3d,f). The spectral shape can be reliably fitted for both cases by a minimum of three peaks, though more components cannot be excluded. All BEs are summarized in Table 1. The components at higher binding energies are assigned to $s p^{3}$ hybridized $\mathrm{C}$ atoms. On the basis of the relative peak areas, the total ratio of $s p^{3}$ to $s p^{2} \mathrm{C}$ atoms is estimated to be as high as $50 \%$ for H/MG/ $\mathrm{Pt}$ and $67 \%$ for H/MG/Ir and is thus much larger than the amount of hydrogenated carbon atoms on our reference graphite surface. Since all investigated substrates are treated with atomic hydrogen under identical conditions, the obtained values indicate that the hydrogenation process is considerably altered by the presence of the underlying TM substrate. In order to get insight into the nature of this effect, we consider in the following how graphene hydrogenation is reflected in the spectra of metal substrates.

Figure 4 shows the Pt and Ir 4f core level spectra for MG/Pt and $\mathrm{MG} / \mathrm{Ir}$ before and after hydrogenation. Before the $\mathrm{H}$ treatment, both $\mathrm{Pt}$ and $\mathrm{Ir}$ 4f spectra consist of the bulk component at higher BEs and the interface component at lower BEs. Their shapes are nearly identical to that from the pristine $\operatorname{Pt}(111)$ and $\operatorname{Ir}(111)$ surfaces (not shown), confirming a weak interaction between MG and these two metal surfaces. Upon hydrogenation, the spectral shape is drastically changed. In the Pt 4f spectrum, the interface component (originally at 70.55 $\mathrm{eV}$ ) is greatly reduced, while a new peak at $71.4 \mathrm{eV}$ appears. Thus, the adsorption-induced shift to higher energies is as large
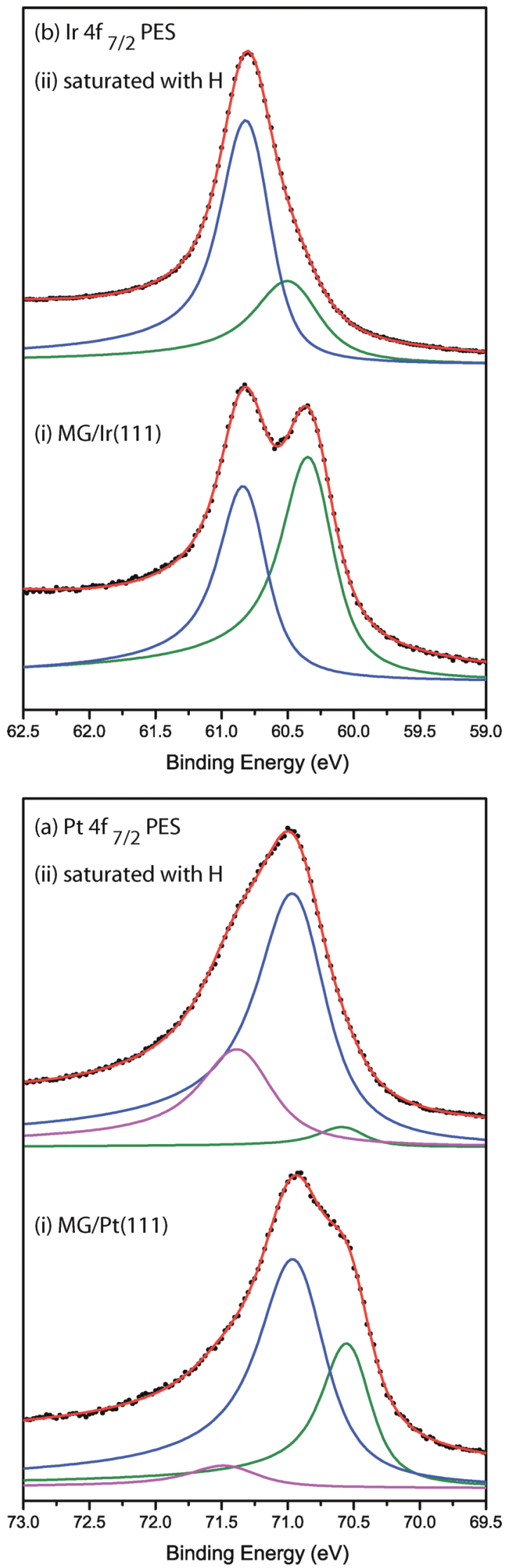

Figure 4. $\mathrm{Pt} 4 \mathrm{f}_{7 / 2}$ PES $(\mathrm{h} v=150 \mathrm{eV})$ and $\operatorname{Ir} 4 \mathrm{f}_{7 / 2}$ PES $(\mathrm{h} v=120 \mathrm{eV})$ of $\mathrm{MG} / \mathrm{Pt}(111)$ and $\mathrm{MG} / \mathrm{Ir}(111)$ before and after saturated $\mathrm{H}$ adsorption.

as $0.85 \mathrm{eV}$. This implies that the $5 \mathrm{~d}$ dangling bonds of the topmost Pt layer are now interacting strongly with the graphene 
layer and the corresponding $5 \mathrm{~d}$ electrons are transferred to the newly formed states resulting in the higher BEs. We have verified that the new component at $71.4 \mathrm{eV}$ is not due to the formation of direct $\mathrm{Pt}-\mathrm{H}$ bonds through hydrogenation of the pristine Pt(111) surface. However, it should be noted that a weak peak at $71.5 \mathrm{eV}$ in the $\mathrm{Pt} 4 \mathrm{f}$ spectrum is visible even before hydrogenation (Figure 4a), probably as a consequence of the $\mathrm{C}-\mathrm{Pt}$ bonding on grain boundaries and other sites with reduced carbon coordination. On Ir, the situation upon hydrogenation is similar (Figure 4b) albeit that the shift of the interface component is smaller than on Pt. Although the spectrum is fitted with only two components, the real situation is probably more complex due to the fact that different $\mathrm{C}$ atoms across the pore form bonds of different strength with the surface Ir atoms. The resulting Ir $4 \mathrm{f}$ spectrum contains a superposition of interface components with BEs that can range from $60.5 \mathrm{eV}$ (low-energy component in our fit) to $60.8 \mathrm{eV}$ (bulk component). Since the original interface component (before hydrogenation) has a $\mathrm{BE}$ of $60.35 \mathrm{eV}$, the adsorption-induced shifts can therefore range from 0.15 to $0.45 \mathrm{eV}$, which is considerably smaller than the shifts in the Pt $4 \mathrm{f}$ spectrum for MG/Pt $(0.85 \mathrm{eV})$.

The values of the BE shifts can be used for estimating strength of the C-TM bonds formed upon graphene hydrogenation. This approach is based on the well-known relation between surface core level shifts and the surface energy difference between the $\mathrm{Z}$ and $\mathrm{Z}+1$ metals, ${ }^{23,24}$ as applied to adsorbate systems. ${ }^{25,26}$ In the framework of the $(Z+1)$ approximation, the adsorbateinduced BE shifts are proportional to the difference in the metaladsorbate bond energies for $\mathrm{Z}$ and $\mathrm{Z}+1$ metals:

$$
\Delta E_{\mathrm{B}}^{\mathrm{Z}}=\sigma\left(E^{\mathrm{Z}, \mathrm{A}}-E^{\mathrm{Z}+1, \mathrm{~A}}\right)
$$

where $\Delta E_{\mathrm{B}}^{Z}$ is the variation in the binding energy of a core electron for the $\mathrm{Z}$ metal atom, $E^{\mathrm{Z}, \mathrm{A}}$ is the energy of the bond between adsorbate atom $\mathrm{A}$ and $\mathrm{Z}$ metal atom and $\sigma$ depends on the local adsorbate-to-substrate geometry. Parameter $\sigma$ can be approximated as $1 / n$ for isolated adsorbates, where $n$ is the number of surface atoms to which an adsorbate bonds, ${ }^{25}$ and can be set to 1 in the case of atomic $\mathrm{H}$ adsorption on graphene. For $\mathrm{Pt}-\mathrm{C}$ and $\mathrm{Ir}-\mathrm{C}$ bonds eq 1 transforms to the following:

$$
\begin{gathered}
\Delta E_{\mathrm{B}}^{\mathrm{Pt}-\mathrm{C}} \approx E(\mathrm{Pt}-\mathrm{C})-E(\mathrm{Au}-\mathrm{C}) \\
\Delta E_{\mathrm{B}}^{\mathrm{Ir}-\mathrm{C}} \approx E(\mathrm{Ir}-\mathrm{C})-E(\mathrm{Pt}-\mathrm{C})
\end{gathered}
$$

Using values of experimental shifts and assuming $E(\mathrm{Au}-\mathrm{C})$ $\approx 0$, we estimate $E(\mathrm{Pt}-\mathrm{C}) \approx 0.85 \mathrm{eV}$ and $E(\mathrm{Ir}-\mathrm{C}) \approx 1.0-1.3$ $\mathrm{eV}$. Therefore, the strength of interaction between the TM substrates and $\mathrm{C}$ atoms of graphene is drastically enhanced upon hydrogenation, indicating a transition from the original weak $\pi$ bonding ( $s p^{2}$ hybridization) to the formation of strong $\sigma$ bonds ( $s p^{3}$ hybridization). We associate this change with destroying the $\mathrm{C}=\mathrm{C}$ double bonds in graphene upon $\mathrm{H}$ adsorption, followed by the engagement of the extra unpaired electrons into the bonding with the substrate.

We return now to the relatively high ratio of $s p^{3}$ hybridized carbons in MG/Pt, and the even higher ratio in MG/Ir. Assuming for the moment that the hydrogenation occurs only on the top side of the $\mathrm{MG}$, it is accompanied by the formation of stronger $\mathrm{Pt}-\mathrm{C}$ respectively $\mathrm{Ir}-\mathrm{C}$ bonds. The most energetically stable configuration should be the graphane-like constellation, where every second $\mathrm{C}$ atom is bound to a hydrogen and buckled upward while the rest form strong bonds with the substrate and are thus buckled downward. This structure has recently been predicted for hydrogen adsorbed on MG/Ir. ${ }^{10}$ It has been found that such bonding to the substrate can occur only on those areas of the surface where every second $\mathrm{C}$ atom is situated above a substrate atom, i.e., in the pores of the MG/Ir moiré structure. The C-substrate distance in these graphane-like areas is then also expected to be somewhat shortened as compared to pristine MG/Ir. Thus, the bonding sites (pores) are the first candidates for the formation of the $\mathrm{H}$ induced graphane-like structures.

According to this picture, a possible assignment for the components in the $\mathrm{C} 1 \mathrm{~s}$ spectra is the following. Peak $\mathrm{C} 1$ (around $284 \mathrm{eV}$ ) is assigned to the $\mathrm{C}$ atoms preserving the $s p^{2}$ coordination. Peak C2 (around $285 \mathrm{eV}$ ) has a BE similar to that of the $\mathrm{C}-\mathrm{H}$ component in the HOPG case and therefore it is assigned to the $\mathrm{C}-\mathrm{H}$ bonds on the elevated nonbonding stripes of graphene (wires). The intensity of peak C3 (around 284.5 $\mathrm{eV}$ ) correlates with the relative area covered by the bonding sites (pores), being higher for MG/Ir than for MG/Pt. Therefore, we associate $\mathrm{C} 3$ with the $s p^{3}$-bonded $\mathrm{C}$ atoms in the pores, both hydrogenated (buckled upward) and bound to the substrate (buckled downward).

We cannot fully exclude the possibility that some of the hydrogen can intercalate under the graphene layer, however, for a number of reasons we find it unlikely: (i) The fully formed graphane would show only $\mathrm{C} s p^{3}$ bonds, yet in our spectra a clear $\mathrm{sp}^{2}$ signal remains at all times. Second, the signature of the measured metal core level spectra is consistent with the increased C-metal bonding. (ii) From PES measurements of metallic clusters (e.g., Ir, Pt, Au) on MG/Ir we observe the same behavior in both the substrate core level as well as the carbon core level, upon cluster formation. ${ }^{27}$ Again, this can be explained by an increased interaction between graphene and the underlying substrate. (iii) On $\mathrm{H} / \mathrm{MG} / \mathrm{Pt}$, no signal consistent with $\mathrm{Pt}-\mathrm{H}$ bonds is observed, as checked by direct hydrogenation of clean $\mathrm{Pt}(111)$. It is interesting to note that intercalation of atomic $\mathrm{H}$ does happen at the MG/SiC interface, where $\mathrm{H}$ atoms form bonds to the topmost $\mathrm{Si}$ atoms, breaking the covalent $\mathrm{Si}-\mathrm{C}$ interaction between substrate and the buffer layer. ${ }^{28}$ In contrast, on noble metals like Pt and Ir, graphene is already preactivated, and the reactive $\mathrm{H}$ atoms can readily stick to it in the pores forming a graphane-like structure, making the lengthy process of intercalation through defects less favorable.

The case of $\mathrm{H} / \mathrm{MG} / \mathrm{Ni}$ is very different from $\mathrm{H} / \mathrm{MG} / \mathrm{Pt}$ and $\mathrm{H} / \mathrm{MG} / \mathrm{Ir}$, because the $\mathrm{MG}-\mathrm{Ni}$ bonds are already strong and short $\left(\sim 2.1 \AA^{20,29}\right)$ and the interface is lattice matched (e.g., no mismatch-related pores and wires). Figure 5 shows the carbon core level and schematics of MG/Ni before and after hydrogenation. The $\mathrm{C} 1 \mathrm{~s}$ spectrum of the initial graphene on Ni(111) consists of a single component (Figure 5a) at $284.8 \mathrm{eV}$, in accordance with earlier studies. ${ }^{21,30}$ The single-component shape of the $\mathrm{C} 1 \mathrm{~s}$ peak is somewhat surprising, because carbon atoms are adsorbed on two distinct sites (on top of $\mathrm{Ni}$ atoms and in the fcc hollows ${ }^{20,21}$ ); it can be explained by strong delocalization of electron density between adjacent atoms in the graphene sheet. An additional tiny component at $283.2 \mathrm{eV}$ is attributed to carbon dissolved in the bulk, and since neither its intensity nor the shape changes after hydrogenation, it can be excluded from the discussion. Upon hydrogenation (Figure 5b) two shoulders (c2 and c3) develop on the opposite sides of the original peak ( $\mathrm{c} 1)$, and their contribution to the total $\mathrm{C}$ 1s signal reaches $45 \%$. (These components are denoted by small letters in order to distinguish this special case from $\mathrm{H} / \mathrm{MG} / \mathrm{Pt}$ and 

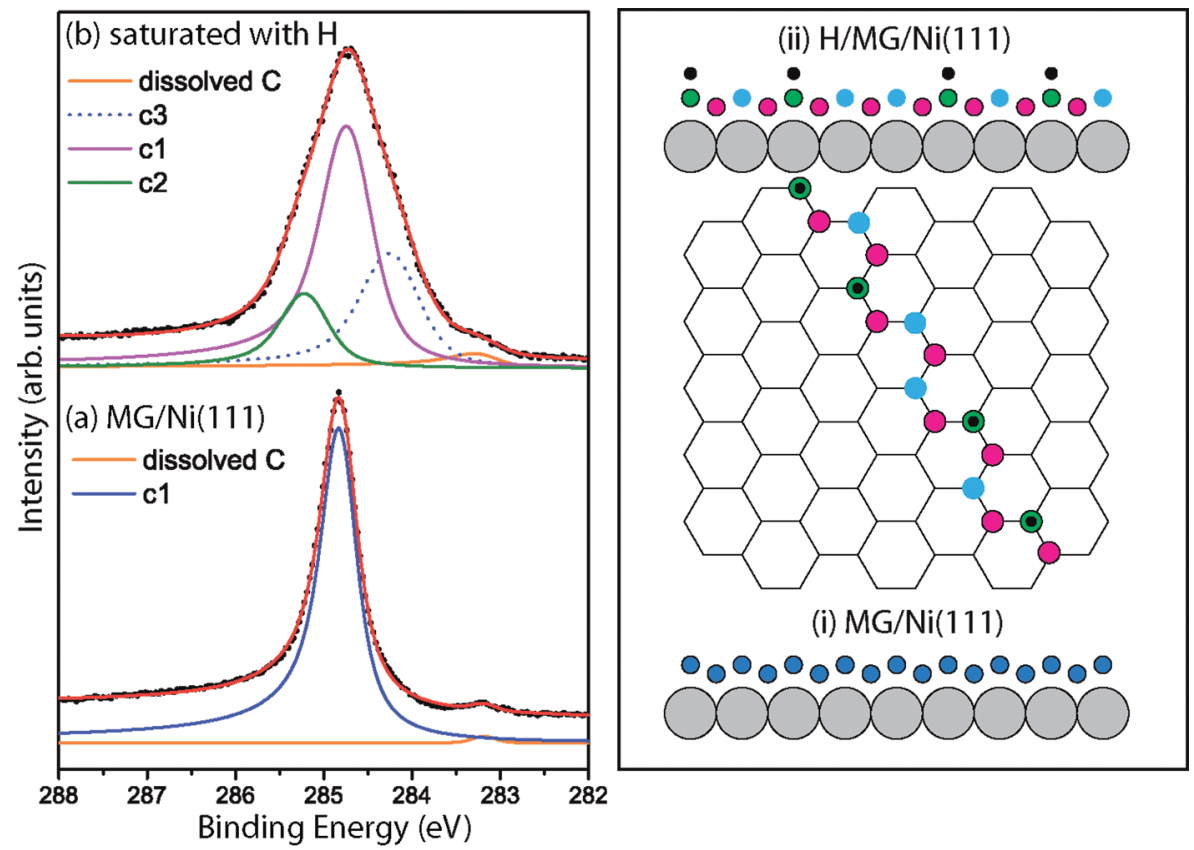

Figure 5. $\mathrm{C} 1 \mathrm{~s}$ PES (hv= $400 \mathrm{eV}$ ) MG/Ni(111) (a) before and (b) after hydrogenation. The schematics show the MG/Ni (i) lateral view before hydrogenation and (ii) lateral and top view after hydrogenation to saturation.

$\mathrm{H} / \mathrm{MG} / \mathrm{Ir}$ ). It is plausible to assign peak $\mathrm{c} 1$ to the $\mathrm{C}$ atoms above the Ni fcc hollow sites, because these atoms are already buckled downward, and cannot attract hydrogen, which sticks only to the upward buckled atoms in the "on top" positions (above the $\mathrm{Ni}$ atoms). ${ }^{31}$ This interpretation is supported by the contribution of $\mathrm{c} 1$ to the total $\mathrm{C} 1 \mathrm{~s}$ intensity (55\%), which is close to the fraction of the $\mathrm{C}$ atoms in the fcc hollow sites $(50 \%)$. By analogy with $\mathrm{H}$ on $\mathrm{HOPG}, \mathrm{MG} / \mathrm{Pt}$, and $\mathrm{MG} / \mathrm{Ir}$, peak $\mathrm{c} 2$ can be associated with the hydrogenated $\mathrm{C}$ atoms in the "on top" positions. Evidently, it is impossible to explain the C 1s spectral shape with the formation of a graphane-like layer, which would then result in just two components ( 1 and $\mathrm{c} 2$ ) of equal intensity. On the contrary, we observe an intense component $\mathrm{c} 3$ at lower $\mathrm{BE}$, which has to be assigned to the non-hydrogenated $\mathrm{C}$ atoms in the "on top" positions.

Our explanation of the fact that only a part of the on-top C atoms can accept hydrogen is the following. The adsorption of individual $\mathrm{H}$ atom on graphene necessarily breaks the $\mathrm{C}=\mathrm{C}$ bond and creates an unpaired electron. Saturating this dangling bond on the same graphene sublattice is energetically unfavorable ${ }^{4}$ and is the reason why meta-dimers (1,3-dihydrogenated ring) are less favorable on graphite (0001). ${ }^{14}$ In the case of MG/Ni the unpaired electron cannot be transferred to the fcc hollow sublattice because of the strong bonding to the underlying $\mathrm{Ni}$, which makes the barrier for new bond formation very high. Therefore, the unpaired electron remains on the nearest neighbors belonging to the same sublattice (positions 3 and 5 in the $\mathrm{C}$ ring assuming the adsorption position is 1 ). The increased electron density on these positions elevates the barrier for the $\mathrm{H}$ adsorption, thus preventing the corresponding on-top $\mathrm{C}$ atoms from forming the $\mathrm{C}-\mathrm{H}$ bonds. The increased electron density on these atoms shifts the corresponding C 1s peak (c3) to lower $\mathrm{BEs}$, in agreement with our experiment. In the schematic drawing of Figure 5 red circles represent the fcc sublattice (c1, prohibited for $\mathrm{H}$ adsorption), while green and blue circles stand for the top sublattice. If green sites are hydrogenated (c2), then blue sites cannot be hydrogenated any more (c3). Evidently, the ratio of sites $\mathrm{c} 1: \mathrm{c} 2: \mathrm{c} 3$ is theoretically limited to $3: 1: 2(\sim 50 \%$, $17 \%$, and $33 \%$ ), but since $\mathrm{H}$ adsorption happens randomly the c2/c3 intensity ratio may be reduced. The experimental intensity ratio of peaks $\mathrm{c} 1, \mathrm{c} 2$, and $\mathrm{c} 3$ are fairly close to the above limit, i.e., $55 \%, 16 \%$, and $29 \%$, respectively.

\section{Conclusions}

Graphene's ability to adsorb atomic hydrogen is strongly dependent on the character of the underlying substrate. Without a reactive substrate, the saturated $\mathrm{H}$ coverage is relatively low. By adsorption of graphene on a reactive metal substrate, the $\mathrm{H}$ uptake value can be increased significantly. At the present hydrogen dosing conditions, the highest values are observed for MG/Ir(111), less for MG/Pt(111), even less for MG/Ni, and the least for the adsorption on bulk graphite. On the latticemismatched MG/Pt and MG/Ir interfaces, graphene is slightly corrugated on the nanometer scale inducing site selectivity for $\mathrm{H}$ adsorption. The more bonding graphene patches (pores) are able to achieve $50 \% \mathrm{H}$ coverage by forming graphane-like structures. This process becomes possible due to the strengthening the $\mathrm{C} 2 \mathrm{p}_{z}$-TM $5 \mathrm{~d}$ bonds, which can involve the unpaired electrons released upon breaking the $\mathrm{C}=\mathrm{C}$ bonds in the course of $\mathrm{H}$ adsorption. The total area of more strongly bonding graphene sites (pores) is higher for MG on Ir than on Pt, thus resulting in the higher $\mathrm{H}$ coverage. The nonbonding graphene patches (wires) are much more passive, and hydrogenation of these sites is expected to be similar to that of HOPG. For the already strongly bound and lattice matched interfaces (MG/Ni), no further bond strengthening is possible, and moreover, the hollow site sublattice is excluded from hydrogenation entirely. A graphane-like structure can therefore not be found and the dangling bonds have to be accommodated on the same sublattice (top sites). This produces a strong reduction in the $\mathrm{H}$ uptake value, where each formed $\mathrm{C}-\mathrm{H}$ bond means the blocking of at least two $\mathrm{C}$ atoms from further hydrogen adsorption.

Acknowledgment. M.L.N. and N.A.V. are grateful for the financial supports from the Swedish Research Council. R.B. and L.H. gratefully acknowledge the financial support from the 
European Research Council under ERC starting grant no. 208344, HPAH. The fruitful discussion with P. Aldenius is much appreciated.

\section{References and Notes}

(1) Novoselov, K. S.; Geim, A. K.; Morozov, S. V.; Jiang, D.; Katsnelson, M. I.; Grigorieva, I. V.; Dubonos, S. V.; Firsov, A. A. Nature 2005, 438, 197.

(2) Morozov, S. V.; Novoselov, K. S.; Katsnelson, M. I.; Schedin, F.; Elias, D. C.; Jaszczak, J. A.; Geim, A. K. Phys. Rev. Lett. 2008, 100, 016602.

(3) Schedin, F.; Geim, A. K.; Morozov, S. V.; Hill, E. W.; Blake, P.; Katsnelson, M. I.; Novoselov, K. S. Nat. Mater. 2007, 6, 652.

(4) Boukhvalov, D. W.; Katsnelson, M. I. J. Phys.: Condsens. Matter 2009, 21, 344205 .

(5) Zhou, J.; Wang, Q.; Sun, Q.; Chen, X. S.; Kawazoe, Y.; Jena, P. Nano Lett. 2009, 9, 3867.

(6) Boukhvalov, D. W.; Katsnelson, M. I.; Lichtenstein, A. I. Phys. Rev. B 2008, 77, 035427.

(7) Boukhvalov, D. W.; Katsnelson, M. I. Phys. Rev. B 2008, 78, 085413

(8) Elias, D. C.; Nair, R. R.; Mohiuddin, M. G.; Morozov, S. V.; Blake, P.; Halsall, M. P.; Ferrari, A. C.; Boukhvalov, D. W.; Katsnelson, M. I.; Geim, A. K.; Novoselov, K. S. Science 2009, 323, 610.

(9) Ferro, Y.; Teillet-Billy, D.; Rougeau, N.; Sidis, V.; Morisset, S.; Allouche, A. Phys. Rev. B 2008, 78, 085417.

(10) Balog, R.; Jørgensen, B.; Nilsson, L.; Andersen, M.; Rienks, E.; Bianchi, M.; Fanetti, M.; Lægsgaard, E.; Baraldi, A.; Lizzit, S.; Sljivančanin, Ž. Besenbacher, F.; Hammer, B.; Pedersen, T. G.; Hofmann, P.; Hornekær, L., Nat. Mater., DOI:10.1038/NMAT2710.

(11) Sofo, J. O.; Chaudhari, A. S.; Barber, G. D. Phys. Rev. B 2007, $75,153401$.

(12) Deng, W.-Q.; Xu, X.; Goddard, W. A. Phys. Rev. Lett. 2004, 92, 166103
(13) Balog, R.; Jørgensen, B.; Wells, J.; Lægsgaard, E.; Hofmann, P.; Besenbacher, F.; Hornekær, L. J. Am. Chem. Soc. 2009, 131, 8744.

(14) Hornekær, L.; Sljivančanin, Ž.; Xu, W.; Otero, R.; Rauls, E.; Stensgaard, I.; Lægsgaard, E.; Hammer, B.; Besenbacher, F. Phys. Rev. Lett. 2006, 96, 156104.

(15) Preobrajenski, A. B.; Ng, M. L.; Vinogradov, A. S.; Mårtensson, N. Phys. Rev. B 2008, 78, 073401.

(16) Wintterlin, J.; Bocquet, M.-L. Surf. Sci. 2009, 603, 1841.

(17) Adams D. L. FitXPS (version 2.12). http://www.sljus.lu.se/download.html (accessed May 28, 2009).

(18) Stöhr, J. NEXAFS Spectroscopy; Springer: Berlin, 1992.

(19) Nikitin, A.; Näslund, L.-Å.; Zhang, Z.; Nilsson, A. Surf. Sci. 2008, $602,2575$.

(20) Bertoni, G.; Calmels, L.; Altibelli, A.; Serin, V. Phys. Rev. B 2004, $71,075402$.

(21) Rusz, J.; Preobrajenski, A. B.; Ng, M. L.; Vinogradov, N. A.; Mårtensson, N.; Wessely, O.; Sanyal, B.; Eriksson, O. Phys. Rev. B 2010, 81,073402 .

(22) Tanuma, S.; Powell, C. J.; Penn, D. R. Surf. Interface Anal. 2005, $37,1$.

(23) Johansson, B.; Mårtensson, N. Phys. Rev. B 1980, 21, 4427.

(24) Johansson, B.; Mårtensson, N. Helv. Phys. Acta 1983, 56, 405.

(25) Tréglia, G.; Desjonquères, M. C.; Spanjaard, D.; Lassailly, Y.; Guillot, C.; Jugnet, Y.; Duc, T. M.; Lecante, J. J. Phys. C: Solid State Phys. 1981, 14, 3463.

(26) Dückers, K.; Prince, K. C.; Bonzel, H. P.; Cháb, V.; Horn, K. Phys. Rev. B 1987, 36, 6292 .

(27) J. Knudsen et al., unpublished work.

(28) Riedl, C.; Coletti, C.; Iwasaki, T.; Zakharov, A. A.; Starke, U. Phys. Rev. Lett. 2009, 103, 246804

(29) Gamo, Y.; Nagashima, A.; Wakabayashi, M.; Terai, M.; Oshima, C. Surf. Sci. 1997, 374, 61.

(30) Grüneis, A.; Kummer, K.; Vyalikh, D. V. New J. Phys. 2009, 11, 073050.

(31) Sha, X.; Jackson, B. Surf. Sci. 2002, 496, 318.

JP106361Y 